\title{
Partial transmit sequence scheme with new phase sequence for PAPR reduction in OFDM systems
}

\begin{abstract}
One of the effective methods used for reducing peak to average power ratio (PAPR) in OFDM systems is partial transmit sequence (PTS). In the conventional PTS (CPTS) several inverse fast Fourier transform (IFFT) operations and complicated calculation to obtain optimum phase sequence, increase the computational complexity of C-PTS. In this paper, we propose a technique to reduce the number of IFFT operations to half at the expense of a slight PAPR degradation. Simulations are performed with QPSK modulation with OFDM signal and Saleh model power amplifier. The effects of digital predistortion (DPD) to increase the linearity and efficiency of the Saleh model power amplifier (PA) are also examined.
\end{abstract}

Keyword: OFDM; Power amplifier; Partial transmit sequence; PAPR; CCDF 